\title{
SENSOR SELECTION FOR OBSERVER FEEDBACK IN TURBOCHARGED SPARK IGNITED ENGINES
}

\author{
Per Andersson, Erik Frisk, and Lars Eriksson
}

\author{
Vehicular Systems, ISY \\ Linköping University, SE-581 83 Linköping, SWEDEN \\ \{peran,frisk,larer\}@isy.liu.se
}

\begin{abstract}
Precise air/fuel control is necessary for low emissions in turbocharged (TC) SI-engines. Observers are often proposed for accurate cylinder air charge estimation in air/fuel controllers. First it is shown using a structural method that the system is locally structurally observable using observer-feedback from any measured state. A systematic method to select observer feedback signals is important as the number of possible sensor combinations reach 680 when 3 sensors are used which could be placed at 17 different locations. Here a systematic method based on observability index is proposed that is able to point out one combination of measured signals as good candidates to observe the states needed for CAC estimation. Finally the results are valid for all TC SI-engines with the same structure but the method is generally applicable. Copyright (C)2005 IFAC
\end{abstract}

Keywords: automotive control, automotive emissions, engine control, engine management, model based control, observability, structural properties

\section{INTRODUCTION}

In spark ignited (SI) engines accurate air/fuel control is essential to successfully reduce the emissions using a three way catalyst (Heywood, 1988; Bauer et al., 1996; Kiencke and Nielsen, 2000; Mondt, 2000). To control the air/fuel-ratio, the mass of fuel to inject is determined given estimates of the cylinder air charge (CAC). As signals involved in the CAC calculation are subjected to noise and the necessity for prediction to achieve good transient control motivates the use of observers for the CAC estimation (Powell et al., 1998; Choi and Hedrick, 1998). Observers have been successfully used for CAC estimation on naturally aspirated engines, but here turbocharged (TC) engines are considered which are more complex and there are considerably more possible sensor locations on TC-engines. Also the use of observers enables the available sensors to be used for observer feedback which enables more information to be extracted regarding the system and perhaps reduce the number of sensors. The use of few sensors is very desirable for the cost sensitive automotive industry.

Observers require feedback from one or more sensors. An important question that arises during the design phase is: - Given a limited number of sensors, what sensors and sensor configurations are the best choice? This question is addressed here. A challenge is that the number of possible sensor combinations virtually explode when more than one sensor is used, which is illustrated in the following example. Here there are 17 possible sensor sources. When two sensors are used there are $\left(\begin{array}{c}17 \\ 2\end{array}\right)=136$ combinations and if three sensors are used then there are $\left(\begin{array}{c}17 \\ 3\end{array}\right)=680$ combinations. Therefore a systematic method is proposed to select what sensor(s) to use or at least reduce the number of possible candidates.

A necessary condition in observer design is that the system is observable from the selected feedback signal(s). It is shown that the system is locally structurally observable from any measured state or function 
of measured states such as air-mass flows. Thus there are a vast number of possible sensor configurations.

In the application, CAC estimation, some states are more important than others. This is taken into account when a systematic method to select sensors for observer feedback is proposed.

\section{ENGINE MODEL}

The observer design relies on a nonlinear mean value engine model of a turbocharged SI-engine, see e.g. Hendricks and Sorensen (1990) and Eriksson et al. (2002). Turbocharged engines are similar with respect to their structure: They have air-filter, compressor, intercooler, throttle, intake manifold, exhaust manifold, turbine, and an exhaust system. At most of these components it is possible to measure pressure and temperature and air-mass flow(s).

The engine modeling methodology is to place control volumes between restrictions (Eriksson et al., 2002) and the physical structure of the turbocharged engine model used is shown in Figure 1 where also some possible sensor locations are shown. In the model, described in detail in Andersson (2005), there are 13 states and 5 inputs.

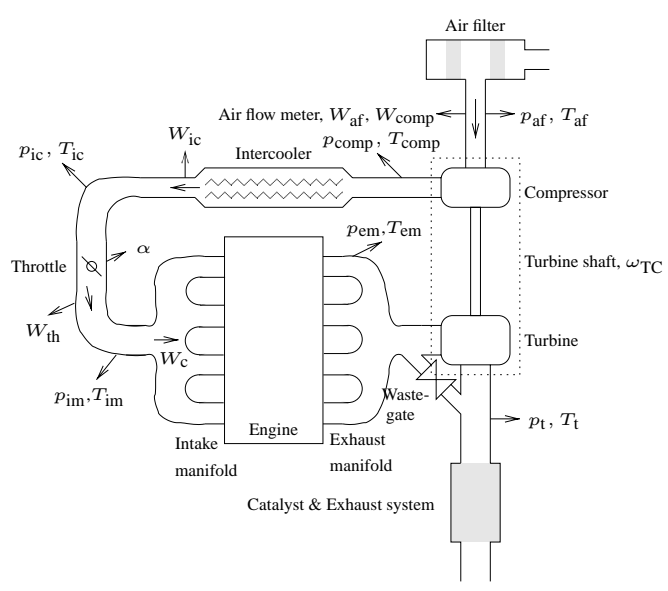

Fig. 1. A schematic of a turbocharged SI-engine. Arrows pointing out of the engine indicate possible sensor placements. Arrows inside the engine indicate air-mass flows, of which the air-mass flow into the cylinders $W_{\mathrm{c}}$ is of particular interest for air/fuel ratio control.

The nomenclature is that pressures are denoted $p$, temperatures $T$, air-mass flows $W$, and the index shows the location. The locations are: air-filter (af), compressor (comp), intercooler (ic), intake manifold (im), exhaust manifold (em), and turbine $(\mathrm{t})$. The turbocharger speed is indicated by $\omega_{\text {TC }}$. Next model inputs, states, and outputs are explained.

\subsection{Inputs}

Inputs to this model are engine speed $N$, throttle plate angle $\alpha$, the desired air/fuel ratio $\lambda$, opening of the wastegate $u_{\mathrm{wg}}$, together with ambient conditions such as pressure $p_{\mathrm{a}}$ and temperature $T_{\mathrm{a}}$.

\subsection{States and State Equations}

The states in the model are six pairs of pressure and temperature states and the turbocharger speed, $\omega_{\mathrm{TC}}$. Now the engine model is described by the following system of differential equations.

$$
\begin{aligned}
& e_{1}: \quad \dot{p}_{\text {af }}=f_{p_{\text {af }}}\left(p_{\text {af }}, T_{\text {af }}, p_{\text {comp }}, \omega_{\mathrm{TC}}, p_{\mathrm{a}}, T_{\mathrm{a}}\right) \\
& e_{2}: \quad \dot{T}_{\text {af }}=f_{T_{\text {af }}}\left(p_{\text {af }}, T_{\text {af }}, p_{\text {comp }}, \omega_{\mathrm{TC}}, p_{\mathrm{a}}, T_{\mathrm{a}}\right) \\
& e_{3}: \quad \dot{p}_{\text {comp }}=f_{p_{\text {comp }}}\left(p_{\text {af }}, T_{\text {af }}, p_{\text {comp }}, T_{\text {comp }}, p_{\text {ic }}, \omega_{\text {TC }}\right) \\
& e_{4}: \quad \dot{T}_{\text {comp }}=f_{T_{\text {comp }}}\left(p_{\text {af }}, T_{\text {af }}, p_{\text {comp }}, T_{\text {comp }}, p_{\text {ic }}, \omega_{\text {TC }}\right) \\
& e_{5}: \quad \dot{p}_{\text {ic }}=f_{p_{\text {ic }}}\left(p_{\text {comp }}, T_{\text {comp }}, p_{\text {ic }}, T_{\text {ic }}, p_{\text {im }}, \alpha\right) \\
& e_{6}: \quad \dot{T}_{\mathrm{ic}}=f_{T_{\mathrm{ic}}}\left(p_{\text {comp }}, T_{\text {comp }}, p_{\mathrm{ic}}, T_{\mathrm{ic}}, p_{\mathrm{im}}, \alpha\right) \\
& e_{7}: \quad \dot{p}_{\text {im }}=f_{p_{\text {im }}}\left(p_{\text {ic }}, T_{\text {ic }}, T_{\text {im }}, p_{\text {im }}, p_{\text {em }}, N, \alpha, \lambda\right) \\
& e_{8}: \quad \dot{T}_{\mathrm{im}}=f_{T_{\mathrm{im}}}\left(p_{\mathrm{ic}}, T_{\mathrm{ic}}, T_{\mathrm{im}}, p_{\mathrm{im}}, p_{\mathrm{em}}, N, \alpha, \lambda\right) \\
& e_{9}: \quad \dot{p}_{\mathrm{em}}=f_{p_{\mathrm{em}}}\left(p_{\mathrm{im}}, T_{\mathrm{im}}, p_{\mathrm{em}}, T_{\mathrm{em}}, p_{\mathrm{t}}, N, \lambda, u_{\mathrm{wg}}, T_{\mathrm{a}}\right) \\
& e_{10}: \quad \dot{T}_{\mathrm{em}}=f_{T_{\mathrm{em}}}\left(p_{\mathrm{im}}, T_{\mathrm{im}}, p_{\mathrm{em}}, T_{\mathrm{em}}, p_{\mathrm{t}}, N, \lambda, u_{\mathrm{wg}}, T_{\mathrm{a}}\right) \\
& e_{11}: \quad \dot{p}_{\mathrm{t}}=f_{p_{\mathrm{t}}}\left(p_{\mathrm{em}}, T_{\mathrm{em}}, p_{\mathrm{t}}, T_{\mathrm{t}}, \omega_{\mathrm{TC}}, u_{\mathrm{wg}}, p_{\mathrm{a}}\right) \\
& e_{12}: \quad \dot{T}_{\mathrm{t}}=f_{T_{\mathrm{t}}}\left(p_{\mathrm{em}}, T_{\mathrm{em}}, p_{\mathrm{t}}, T_{\mathrm{t}}, \omega_{\mathrm{TC}}, u_{\mathrm{wg}}, p_{\mathrm{a}}\right) \\
& e_{13}: \quad \dot{\omega}_{\mathrm{tc}}=f_{\omega_{\mathrm{TC}}}\left(p_{\mathrm{af}}, T_{\mathrm{af}}, p_{\text {comp }}, p_{\mathrm{em}}, T_{\mathrm{em}}, p_{\mathrm{t}}, \omega_{\mathrm{TC}}\right)
\end{aligned}
$$

\subsection{Measured Signals}

Measured signals are outputs of the system which are states and/or functions of states and inputs:

$$
y=g(x, u)
$$

An example of a function of states and inputs is the measured air-mass flow after the air-filter which can be expressed as a function of the pressure after the airfilter, a constant parameter $\mathcal{H}_{\mathrm{af}}$, and the inputs $p_{\mathrm{a}}$ and $T_{\mathrm{a}}$ :

$$
W_{\text {af }}=g(\underbrace{p_{\text {af }}}_{x}, \underbrace{p_{\mathrm{a}}, T_{\mathrm{a}}}_{u})=\sqrt{\frac{p_{\mathrm{a}}\left(p_{\mathrm{a}}-p_{\mathrm{af}}\right)}{\mathcal{H}_{\mathrm{af}} T_{\mathrm{a}}}}
$$

Thus it depends only on one state, the pressure after the air-filter. For the other possible air-mass flows in the intake side the state dependencies are as follows:

\section{Compressor air-mass flow}

$W_{\text {comp }}=g\left(p_{\text {af }}, T_{\text {af }}, p_{\text {comp }}, \omega_{\mathrm{TC}}\right)$

Intercooler air-mass flow $W_{\text {ic }}=g\left(p_{\text {comp }}, T_{\text {comp }}, p_{\text {ic }}\right)$ Throttle air-mass flow $W_{\text {th }}=g\left(p_{\text {im }}, p_{\text {ic }}, T_{\text {ic }}\right)$. 
Available Sensor Signals and Sensor Dynamics All states are considered to be measurable and functions of states and inputs such as air-mass flows are only measurable on the intake side. One practical consideration is necessary for temperature sensors as their time constant is in the order of several seconds compared to the considerably faster pressure sensors and airmass flow sensors. Therefore the system is augmented with temperature sensor dynamics for each temperature sensor (Chevalier et al., 2000):

$$
\dot{T}_{\text {sensor }}=\frac{1}{\tau}\left(T-T_{\text {sensor }}\right)
$$

The set of sensors considered here are:

$$
\begin{aligned}
\mathcal{Y}=\{ & \left\{p_{\text {af }}, T_{\text {af }}, p_{\text {comp }}, T_{\text {comp }}, p_{\text {ic }}, T_{\text {ic }}, p_{\text {im }}, T_{\text {im }},\right. \\
& \left.p_{\text {em }}, T_{\text {em }}, p_{\mathrm{t}}, T_{\mathrm{t}}, \omega_{\text {TC }}, W_{\text {af }}, W_{\text {comp }}, W_{\text {ic }}, W_{\text {th }}\right\}
\end{aligned}
$$

Note that all states are considered measurable, but for each measured temperature the system has to be augmented with one state.

\section{OBSERVABILITY}

Before an observer is designed, it must be determined whether the system is observable and as the system is nonlinear, this is not an easy task. One method to show that the system is at least locally observable is to linearize the equation system in Section 2.2 in stationary points and then use linear theory to determine observability. Given that $f_{x}(x, u)$ is the partial derivative of $f(x, u)$ w.r.t. $x,\left(x_{0}, u_{0}\right)$ is a stationary point, then the linearized system matrices $A, B, C$ and $D$ are defined as follows:

$$
\begin{array}{ll}
A=f_{x}\left(x_{0}, u_{0}\right) & B=f_{u}\left(x_{0}, u_{0}\right) \\
C=g_{x}\left(x_{0}, u_{0}\right) & D=g_{u}\left(x_{0}, u_{0}\right)
\end{array}
$$

The linearized system can now be written as

$$
\dot{x}=A x+B u
$$

and the measured signal(s)

$$
y=C x+D u
$$

Measured signals $y$ are selected from the set $\mathcal{Y}$. Introduce the standard notation

$$
\mathcal{O}_{\rho}=\left[\begin{array}{c}
C \\
C A \\
\vdots \\
C A^{\rho-1}
\end{array}\right]
$$

where the matrix $\mathcal{O}$ without index means $\rho=n$, i.e. $\mathcal{O}=\mathcal{O}_{n}$. Then the system is observable if and only if $\mathcal{O}$ has full column rank (Kailath, 1980, p. 81). Unfortunately, the linearized system matrix $A$ is illconditioned due to large differences in time-constants in the engine dynamics. This makes it hard to numerically determine $\mathcal{O}$ as it involves taking $A$ to the power of 12 .

Definition 1. (Observability index). The observability index is defined as the smallest positive integer $\rho$ such that $\mathcal{O}_{\rho}$ has full rank.

\subsection{Observability Index of A Subset of States}

Cylinder air charge estimation depends on $p_{\mathrm{im}}, T_{\mathrm{im}}$, and $p_{\mathrm{em}}$ (Andersson and Eriksson, 2004), which are only 3 out of 13 states and thus the question is asked whether there are combinations of measured signals that better observe these variables. We therefore use observability index of only a part of the state vector as a metric to evaluate sensor configurations. The metric is further discussed in Section 5.

We start by partitioning the state vector $x$ into $x=$ $\left[\begin{array}{l}x_{1} \\ x_{2}\end{array}\right]$, where $x_{1}$ are the states we want to observe. The vector $x_{1}$ has $n_{1}$ states, $x_{2}$ has $n_{2}$ states, and $n_{1}+$ $n_{2}=n$. Now the columns of the observability matrix $\mathcal{O} \in \mathbb{R}^{m \times n}$ are rearranged into $\mathcal{O}_{n}=\left[\begin{array}{ll}\mathcal{O}_{n}^{1} & \mathcal{O}_{n}^{2}\end{array}\right]$, where $\mathcal{O}_{n}^{1} \in \mathbb{R}^{m \times n_{1}}$ and $\mathcal{O}_{n}^{2} \in \mathbb{R}^{m \times n_{2}}$. After the rearrangement the first $n_{1}$ columns in $\mathcal{O}$ corresponds to the states in $x_{1}$. Next observability of a subset of states and its associated observability index are defined.

Definition 2. (Observability of a set of states $x_{1}$ ). The state vector $x_{1}$ is observable if and only if $\mathcal{O}_{n}=$ $\left[\mathcal{O}_{n}^{1} \mathcal{O}_{n}^{2}\right]$ is 1 -full rank (Terrell, 2001) w.r.t. the $n_{1}$ first columns, i.e.

$$
\operatorname{rank} \mathcal{O}_{n}=n_{1}+\operatorname{rank} \mathcal{O}_{n}^{2}
$$

Using this definition we can also define observability index for a subset of the states.

Definition 3. (Observability index of a set of states $\left.x_{1}\right)$ Let $(C, A)$ be an observable pair, then the observability index w.r.t. $x_{1}$ is the smallest positive integer $\rho$ such that $\mathcal{O}_{\rho}=\left[\mathcal{O}_{\rho}^{1} \mathcal{O}_{\rho}^{2}\right]$ is 1 -full rank w.r.t. the $n_{1}$ first columns, i.e.

$$
\operatorname{rank} \mathcal{O}_{\rho}=n_{1}+\operatorname{rank} \mathcal{O}_{\rho}^{2}
$$

As can be seen in Definition 3 it is possible that fewer differentiations, and hence lower powers of the ill-conditioned $A$ will be required, as it is now only required that the rank of $\mathcal{O}_{\rho}$ is at least $n_{1}$. Since fewer differentiations are needed than before the numerical problems are not as severe for the partial observability problem.

\subsection{Structural Observability}

Structural observability makes it possible to avoid the numerical problems with high powers of $A$ in $\mathcal{O}_{n}$. Structural observability only considers the structure of the model and thus only provides a necessary condition for observability. However, for physically based models it is reasonable to assume that structural observability also implies analytical observability (Lin, 1974). 
Lin (1974) introduced the concept of structure to analyze controllability of linear systems. The approach is based on the assumption that a qualitative property such as controllability is determined by the structure of the model, rather than the values of the actual system parameters. Structure here means the zero/nonzero structure of the model matrices $(A, B, C, D)$.

A dual formulation of the controllability definition in (Lin, 1974) gives the following definition of structural observability.

Definition 4. (Structural observability). The pair $(C, A)$ is structural observable if there exists an observable pair $\left(C_{0}, A_{0}\right)$ with the same structure.

Structural rank, sometimes referred to as generic rank or normal rank, of a matrix $A$ is here defined as the maximum rank possible for any matrix with the same structure as $A$. Then it is tempting to think that a pair $(C, A)$ is structurally observable if and only if the observability matrix has full structural rank. However, this is not true (Shields and Pearson, 1976; Lin, 1974) and we will use the following result from (Shields and Pearson, 1976):

Theorem 1. (Structural observability). Let $A \in \mathbb{R}^{n \times n}$ and $C \in \mathbb{R}^{m \times n}$. Then the pair $(C, A)$ is structurally observable if and only if the following $\left[n^{2}+n(m-\right.$ 1)] $\times n^{2}$ matrix has structural rank $n^{2}$.

$$
\left[\begin{array}{cccccc}
I & -A & 0 & \cdots & 0 & 0 \\
0 & I & -A & \cdots & 0 & 0 \\
\vdots & \vdots & \vdots & & \vdots & \vdots \\
0 & 0 & 0 & \cdots & I & -A \\
0 & 0 & 0 & \cdots & 0 & C \\
0 & 0 & 0 & \cdots & C & 0 \\
\vdots & \vdots & \vdots & & \vdots & \vdots \\
0 & 0 & C & \cdots & 0 & 0 \\
0 & C & 0 & \cdots & 0 & 0 \\
C & 0 & 0 & \cdots & 0 & 0
\end{array}\right]
$$

The structural rank of a matrix can be efficiently computed using graph theoretical algorithms for matchings in bipartite graphs. In Matlab, the structural rank is computed using the dmperm command.

\section{OBSERVABILITY OF THE ENGINE MODEL}

The first question to answer is whether the engine model is observable using feedback from $\mathcal{Y}$. As there are numerical difficulties when the analytic observability matrix is computed the structural approach is taken instead.

Looking at the structure of the engine model one can verify that the linearized system only has two structures over the engines entire operating region. The cause of the structural change is that for low pressure ratios over the throttle the pressure and temperature states before the throttle do not depend on the intake manifold pressure.

Below all nonzero elements of the A-matrix are shown using an $X$, and the elements that can be zero in one of the two structures are marked using parentheses.

\begin{tabular}{c|ccccccccccccc} 
Eqn. & $p_{\text {af }}$ & $T_{\text {af }}$ & $p_{\text {comp }}$ & $T_{\text {comp }}$ & $p_{\text {ic }}$ & $T_{\text {ic }}$ & $p_{\text {im }}$ & $T_{\text {im }}$ & $p_{\text {em }}$ & $T_{\text {em }}$ & $p_{\mathrm{t}}$ & $T_{\mathrm{t}}$ & $\omega_{\mathrm{TC}}$ \\
\hline$e_{1}$ & $X$ & $X$ & $X$ & 0 & 0 & 0 & 0 & 0 & 0 & 0 & 0 & 0 & $X$ \\
$e_{2}$ & $X$ & $X$ & $X$ & 0 & 0 & 0 & 0 & 0 & 0 & 0 & 0 & 0 & $X$ \\
$e_{3}$ & $X$ & $X$ & $X$ & $X$ & $X$ & 0 & 0 & 0 & 0 & 0 & 0 & 0 & $X$ \\
$e_{4}$ & $X$ & $X$ & $X$ & $X$ & $X$ & 0 & 0 & 0 & 0 & 0 & 0 & 0 & $X$ \\
$e_{5}$ & 0 & 0 & $X$ & $X$ & $X$ & $X$ & $X)$ & 0 & 0 & 0 & 0 & 0 & 0 \\
$e_{6}$ & 0 & 0 & $X$ & $X$ & $X$ & $X$ & $X$ & 0 & 0 & 0 & 0 & 0 & 0 \\
$e_{7}$ & 0 & 0 & 0 & 0 & $X$ & $X$ & $X$ & $X$ & $X$ & 0 & 0 & 0 & 0 \\
$e_{8}$ & 0 & 0 & 0 & 0 & $X$ & $X$ & $X$ & $X$ & $X$ & 0 & 0 & 0 & 0 \\
$e_{9}$ & 0 & 0 & 0 & 0 & 0 & 0 & $X$ & $X$ & $X$ & $X$ & $X$ & 0 & 0 \\
$e_{10}$ & 0 & 0 & 0 & 0 & 0 & 0 & $X$ & $X$ & $X$ & $X$ & $X$ & 0 & 0 \\
$e_{11}$ & 0 & 0 & 0 & 0 & 0 & 0 & 0 & 0 & $X$ & $X$ & $X$ & $X$ & $X$ \\
$e_{12}$ & 0 & 0 & 0 & 0 & 0 & 0 & 0 & 0 & $X$ & $X$ & $X$ & $X$ & $X$ \\
$e_{13}$ & $X$ & $X$ & $X$ & 0 & 0 & 0 & 0 & 0 & $X$ & $X$ & $X$ & 0 & $X$
\end{tabular}

Using Theorem 1 it can be verified that the linearized engine model is structurally observable from any single sensor signal. Thus the system is locally structurally observable using one feedback from $\mathcal{Y}$ and as $\mathcal{Y}$ includes all states the system is locally structurally observable with feedback from any measured state. This result is valid for all TC SI-engines with the same structure.

\section{SIGNAL SELECTION}

To determine what feedback signal(s) or combination(s) of feedback signals to use it is necessary to choose a selection metric and here systematic methods to choose feedback signal(s) are used. The metrics are based on observability index.

Two scenarios are studied: Signal selection for best observability of the total system and signal selection for best CAC observability.

\subsection{Signal Selection for Best System Observability}

When an arbitrary signal in $\mathcal{Y}$ is used for observer feedback the system is locally structurally observable, as shown in Section 3.2. Given that more than one signal from $\mathcal{Y}$ is selected, is it then possible to find a combination of measured signals that is most suitable, i.e. results in a minimized observability index? Consider $m>1$ feedback signals which result in a $C$ matrix with $m$-rows. The total number of rows in $\mathcal{O}$ still have to be at least $n$, in order for the rank to be $n$. The introduction of more than one measured signal does not reduce the number of necessary rows in $\mathcal{O}$. However the lower bound of the observability index is inversely proportional to the number of feedback signals $m$ :

$$
\rho \geq\left\lceil\frac{n}{m}\right\rceil
$$

Consequently, for the total system the lower bound of the observability index depends on the number of 
system states. As slow sensors introduce additional states it is better to use fast sensors. Further, a specific combination of feedbacks that would give a minimized observability index can not be found using this metric as it is only possible to give a lower bound of the observability index.

\subsection{Signal Selection for Best CAC Observability}

The primary objective in engine air/fuel control is to observe the states necessary for CAC estimation and the states involved in the CAC calculation are $p_{\mathrm{im}}, T_{\mathrm{im}}$, and $p_{\text {em }}$. The criterion for sensor selection is thus to minimize the observability index, according to Definition 3 , for the subset of states $x_{1}=\left\{p_{\mathrm{im}}, T_{\mathrm{im}}, p_{\mathrm{em}}\right\}$. Here this method is illustrated for three feedback signals, which is a case where there are $\left(\begin{array}{c}17 \\ 3\end{array}\right)=680$ combinations.

When three signals are selected from $\mathcal{Y}$ for observer feedback and the observability index for the subset of states $x_{1}$ is evaluated the result are three interesting groups of feedback signals which all have observability index less or equal to 4 . These are summarized in Table 1.

Table 1. Observability index for the subset $x_{1}=\left\{p_{\mathrm{im}}, T_{\mathrm{im}}, p_{\mathrm{em}}\right\}$ using three feedback signals.

\begin{tabular}{cccc}
\hline Index & Signal 1 & Signal 2 & Signal 3 \\
\hline 2 & $p_{\text {im }}$ & $T_{\text {im }}$ & $p_{\text {em }}$ \\
3 & $p_{\text {ic }}$ & $p_{\text {im }}$ & $T_{\text {im }}$ \\
3 & $p_{\text {im }}$ & $T_{\text {im }}$ & $W_{\text {th }}$ \\
3 & $p_{\text {im }}$ & $p_{\text {em }}$ & $T_{\text {em }}$ \\
4 & $p_{\text {im }}$ & $p_{\text {em }}$ & $p_{\mathrm{t}}$ \\
4 & $T_{\text {im }}$ & $p_{\text {em }}$ & $T_{\text {em }}$
\end{tabular}

The results from Table 1 are:

- Only one combination with index 2 . The obvious selection to measure the signals in $x_{1}$ is the best choice, even though it includes one additional state for the sensor dynamics. A practical aspect is that the exhaust manifold pressure is hard to measure.

- Two combinations with index 3. One of them is the combination $p_{\text {ic }}, p_{\text {im }}$, and $T_{\mathrm{im}}$ which are normally measured signals on TC SI-engines.

- Two combinations with index 4 , which both include temperatures.

In the cases above the highest power of $A$ is three, and therefore the numerical problems are avoided. Thus the analytical method is able to produce a very short list of good candidates. As the underlying engine model has physically based parameters it is reasonable to assume that this result is valid for all TC SI-engines with the same structure.

\section{FUTURE WORK}

In order to evaluate the observability index for a subset of states for fewer feedback signals, it is not possible to use an analytic method as has been done here. This is because with fewer measurements, the matrix $A$ in the observability matrix $\mathcal{O}$ needs to be raised to higher powers. The ill-conditioning of matrix $A$ makes the computations numerically uncertain. Therefore it would be beneficial to study structural observability and observability index also for a subset of states.

\section{CONCLUSIONS}

The problem of selecting signals for observer feedback has been studied. Using a structural method it is possible to show that the studied system is locally structurally observable from any measured state or combination of states. A metric, observability index, is used to aid the selection of what signals that are most suitable for observer feedback. Two scenarios are considered: The entire system and a subset of the system which is important for CAC estimation.

In the first scenario, observability of the entire system, it is best to use sensors which do not require the system to be augmented with sensor dynamics. When the system is augmented with sensor dynamics the minimum number of required differentiations increase and hence the observability index.

In the second scenario, when the application cylinder air charge estimation is considered, only a fraction of the state space is required to determine the cylinder air charge. Therefore the observability index of this subset of states is used to evaluate which feedback signal and/or combination of signals that observes this subset best. When three sensors are used only 5 configurations of 680 possible reach an observability index less or equal to 3 . One unique combination is best and reaches the observability index 2 . It is the obvious combination of measuring $p_{\mathrm{im}}, T_{\mathrm{im}}$, and $p_{\mathrm{em}}$. Even though it requires the system to be augmented with one temperature sensor dynamics it is the best choice. Also the normally measured $p_{\mathrm{ic}}, p_{\mathrm{im}}$, and $T_{\mathrm{im}}$ is a very good combination with observability index 3 .

Thus the proposed method provides the observer designer with valuable information during the sensor selection. An advantage is that no signal-to-noise ratios etc. are necessary inputs to the method. Finally the method is generally applicable and the results are valid for all turbocharged SI-engines with the same structure.

\section{ACKNOWLEDGMENTS}

This work is supported by Swedish Agency for Innovation Systems and Fiat-GM Powertrain Sweden. 


\section{REFERENCES}

Andersson, Per (2005). A Component Based Mean Value Engine Model of a Turbocharged SIEngine. Technical report. Vehicular Systems, Department of Electrical Engineering. Linköpings Universitet.

Andersson, Per and Lars Eriksson (2004). Cylinder Air Charge Estimator in Turbocharged SIEngines. Society of Automotive Engineers. SAE Technical Paper No. 2004-01-1366.

Bauer, Horst, Cypra, Arne, Beer, Anton and Bauer, Hans, Eds. (1996). Bosch Automotive Handbook. 4 ed.. Robert Bosch Gmbh.

Chevalier, Alain, Christian Winge Vigild and Elbert Hendricks (2000). Predicting the port air mass flow of SI engines in air/fuel ratio control applications. In: Electronic Engine Controls. SP-1500. SAE Technical Paper No. 2000-01-0260.

Choi, Seibum B. and J. Karl Hedrick (1998). An Observer-Based Controller Design Method for Improving Air/Fuel Characteristics of Spark Ignition Engines. IEEE Transactions on Control Systems Technology 8(3), 325-334.

Eriksson, Lars, Lars Nielsen, Jan Brugård, Johan Bergström, Fredrik Pettersson and Per Andersson (2002). Modeling of a Turbocharged SI Engine. Annual Reviews in Control 26(1), 129-137.

Hendricks, E. and S. C. Sorensen (1990). Mean Value Modelling of Spark Ignition Engines. SAE Technical Paper No. 900616.

Heywood, John B. (1988). Internal Combustion Engine Fundamentals. McGraw-Hill International Editions.

Kailath, Thomas (1980). Linear Systems. PrenticeHall.

Kiencke, Uwe and Lars Nielsen (2000). Automotive Control Systems. For Engine, Driveline, and Vehicle. Springer-Verlag.

Lin, Ching-Tai (1974). Structural controllability. IEEE Transactions on Automatic Control 19(3), 201208.

Mondt, J.R. (2000). Cleaner Cars. The History and Technoloby of Emission Control Since the 1960s. SAE International.

Powell, J. David, N.P Fekete and Chen-Fang Chang (1998). Observer-based air-fuel control. Control Systems Magazine vol. 18(no. 5), pages 7283.

Shields, R.W. and J.B. Pearson (1976). Structural Controllability of Multiinput Linear Systems. IEEE Transactions on Automatic Control 21(2), 203-212.

Terrell, William J. (2001). Local Observability of Nonlinear Differential-Algebraic Equations (DAEs) From the Linearization Along a Trajectory. IEEE Transactions on Automatic Control. 